\title{
The Programmable Synergetic Controller
}

\author{
Xiang Sun ${ }^{1,2}$, Xiaojie Zhao ${ }^{1,2, *}$ and Yongwei Tang ${ }^{1,2}$ \\ ${ }^{1}$ Shandong Computer Science Center (National Supercomputer Center in Jinan), Jinan 250014, China \\ ${ }^{2}$ Shandong Provincial Key Laboratory of Computer Networks, Jinan 250014, China \\ ${ }^{*}$ Corresponding author
}

\begin{abstract}
A new type of Programmable Synergetic Controller (PSC) is introduced in this paper. The synergetic tasks can be transmitted rapidly and efficiently each other by it's embedded concurrent multiple communication modular with the streamlined synergetic information coding system in this controller. According to control goals, the relevant controllers can be organized to work cooperatively spontaneously by the synergetic scheduling management and synergetic control algorithm program in the embedded system software, and can cooperate with each other for the synergetic tasks. In addition, the PSC also has special functions of remote reprogramming and debugging online. As the aspect of hardware, the PSC provides multiple power conversion and management, wireless and wired communication interface, mass data storage, various input / output interfaces and expansion capabilities.
\end{abstract}

Keywords—synergetic controller; collaboration; programmable; controller; PLC

\section{INTRODUCTION}

The Programmable Logic Controller (PLC) has been used widely in various fields and automatic control systems. As the applications are becoming larger and larger, the safety and reliability requirements getting higher and higher, the system structure, measurement and control being more and more complex, not only a single programmable controller cannot be suitable for the applications in these systems, the distributed control system cannot meet the needs well which are composed of many controllers.

The Programmable Synergetic Controller (PSC)[1] introduced in this paper can be improved in many aspects of system reliability, real-time, adaptive ability and so on compared with the conventional programmable controller. In particular, the PSC can realize autonomous control, and can work autonomously with other PSC to achieve the collaborative measurement and control across sites or regions, and even across system. The PSC can be used to simple applications that are accomplished from a single or a few controllers, and to large complex application systems that require lot of, even thousands of controllers, to work together.

\section{HARDWARE STRUCTURE OF THE COOPERATIVE PROGRAMMABLE CONTROLLER}

\section{A. The Block Diagram of CPC Hardware Structure}

The modular structure has been adopted in this Programmable Synergetic Controller, see Fig. 1. There are the main CPU module embedded by the synergetic control algorithm, the mass data storage module, the multiple concurrent communication module, the multiple power supply conversion and management module, the built-in input and output interface, the expansion of input and output interface.

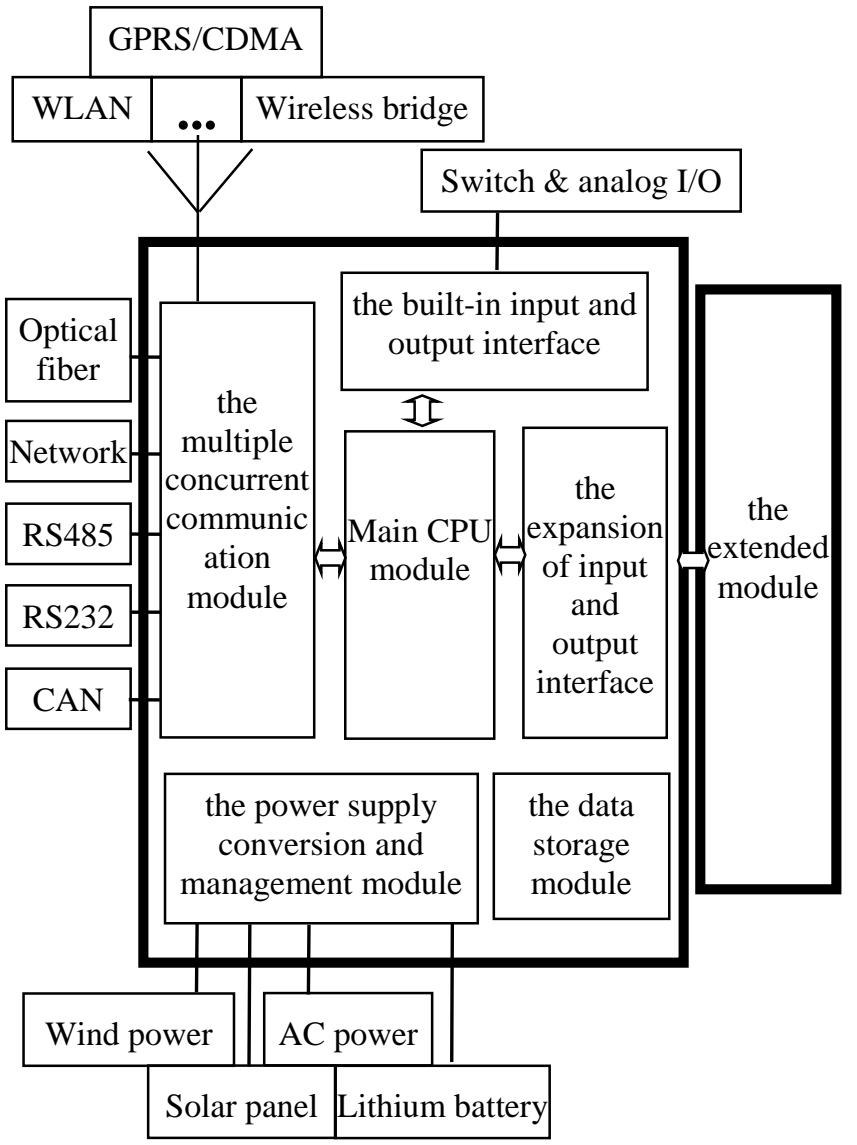

FIGURE I. THE BLOCK DIAGRAM OF CPC HARDWARE STRUCTURE

\section{B. The Main CPU Module}

It is the core of the PSC that main CPU module embedded with the built-in RAM and Flash Memory. The flash memory is divided into three program blocks. The one block of the flash memory, called System Program Area (SPA), to store synergetic control algorithms and system programs. The others called User Program Areas (UPA), are used to store the user programs which can be remote reprogrammed and debugging online.

The user program in one of the UPA could be selected to run in the main CPU module, another UPA can be downloaded at the same time. The synergetic control algorithm and system 
program are always running, and it dynamically manages the other modules of the PSC, and performs the synergetic tasks scheduling, adjudicating, executing and so on.

\section{The Concurrent Multiple Communication Module}

The Concurrent multiple communication module is equipped with two parts of wire communication and wireless communication ${ }^{[2]}$. There are many different types of interfaces and communication protocol in the part of wired communications for connecting external devices or CPC with wire communication interfaces, such as RS232, RS485, CAN, networking (RJ45), fiber optic cables, etc. The wireless communication part including GPRS, CDMA, 3G, 4G, WIFI, wireless network, wireless bridge, microwave and other optional wireless communication plug-in, can be connected to any equipment, Internet, LAN or CPC with the same communication interface.

\section{The Power Supply Transformation and Management Module}

The multiple power supply transformation and management module can not only provide stable power supply to the PSC, but also provide DC5V and DC24V power supply for external sensors and other instruments. According to program settings, the power manager can select and change one or more automatically if the external power interface link to a variety of sources power, such as AC power, small wind power, solar pan, and so on. When the all available external sources power fails, the external battery is switched priority to power supply immediately. The internal battery should be powered only when the external battery is low. In addition, the power monitoring and protection function can provide the current power status information to the CPU module at any time, and send alarm information when necessary.

\section{E. The Input and Output Module}

The input and output module is the interface of the PSC for the external perception and control, including multiple channel analog input and output ports, multiple channel switching input and output ports, and multiple channel pulse input and output ports, audio and video interface and so on. If the input and output interfaces are not enough for the application, the PSC can also provide an extended interface to connect one or more various type extended input and output modules.

The analog input and output ports provided 0 to $5 \mathrm{~V}, 0 \sim 10 \mathrm{~V}$, $-5 \sim+5 \mathrm{~V}, \quad-10 \sim+10 \mathrm{~V}, \quad 0 \sim 20 \mathrm{~mA}, \quad 4 \sim 20 \mathrm{~mA}, \quad-10 \sim+10 \mathrm{~mA}$, 20 +20mA, DC5V0 20KHz and other standard signals. All analog input signals are sent to the main CPU module after AD conversion, and the analog output data is sent to the port after DA conversion. DC0 5V, 0 10V, 0 24V, AC5V, 10V, 24V, $110 \mathrm{~V}, 220 \mathrm{~V}$, and relay passive contacts, SCR switches, can be selected from the switch input and output ports. Pulse input and output port, provided pulse count, PWM, PFM, sine wave and others.

\section{THE EMBEDDED SOFTWARE WITH COOPERATIVE PROGRAMMABLE CONTROLLER}

There are two kinds of software running in the PSC at the same time, one is the system software, and the other is user program. The system software mainly actualizes the synergetic control algorithm, concurrent communication, user program management and coordination among modules, etc. The user program is programmable online to perform user specified functions according to requirements of the applications. What is different with the traditional PLC, the PSC programming language adds a series of cooperation and related operations, for example, to obtain a physical quantity or parameters that cannot be collected by itself, to adjust the parameters of another PSC, allows other PSC read it's data, to complete some tasks with other PSC cooperatively, and so on and so forth.

\section{A. The Synergetic Control Algorithm}

Firstly, the dynamic synergetic information table is created by the synergetic management program in the system software, and the synergetic information of each node PSC in the application system is collected and registered, and the real-time maintenance is carried out according to the operation of the application system.

If the PSC is a sponsor of the synergetic tasks, on the basis of the synergetic information table, it will be targeted to send synergetic requests with its own needs. And it will wait for the participation of the other related PSC, select the partner, hand out the synchronous sequence after the handshake with each other, and perform the synergetic tasks as requested.

If the PSC is a participant, according to its ability to provide synergy, with the synergetic information table, it will undertake the synergetic invitation of the relevant PSC, reply to the sponsor. Finally, it will perform the synergetic tasks with the synchronous timing sequence provided by the sponsor, after a handshake signal was received.

\section{B. The Discriminant of Synergetic Request}

When it is unable to complete its tasks with its own capabilities, the PSC need to send out synergetic requests and invite other PSC to participate. When it has the extra ability to help other PSC to participate in its synergetic tasks, the PSC can undertake the synergetic requests of the other PSC. So, how to determine whether or not it will send or undertake a synergetic requests, the PSC needs to calculate the value with the synergetic request discriminant.

$$
\begin{gathered}
\mathrm{E}=\mathrm{E}_{0} * \mathrm{E}_{1} \\
\mathrm{E}_{0}=\prod \mathrm{A}_{\mathrm{i}} \\
\mathrm{E}_{1}=1 / \mathrm{n} \square \mathrm{Q}_{\mathrm{i}} * \mathrm{~B}_{\mathrm{i}}
\end{gathered}
$$

In (1), E is a value of the synergetic discriminant which each synergetic task has its own, consisting of E0 and E1. If the synergetic task could be passed, E0 is a necessary condition, and E1 is a sufficient condition. In (2), Ai is a single vote veto factor, which takes 1 when the event allows, otherwise takes 0 values. In (3), $\mathrm{Bi}$ is the occurrence probability of the element affecting the event, taking the value between 0 to 1 , Qi is its weight, and also taking the value of 0 to 1 . 
For each synergetic task, the relevant $\mathrm{Ai}, \mathrm{Bi}$, and Qi should be determined in advance. The $\mathrm{Ai}, \mathrm{Bi}$, and $\mathrm{Qi}$ are not the same for different synergetic tasks, which are determined by the user application according to the specific application.

A threshold Et is set for each synergetic discriminant. When the PSC is a sponsor of the synergetic task and E is greater than Et, the synergetic task is generated and the synergetic request will be sent, otherwise the synergetic task will be ignored, see Fig. 2.

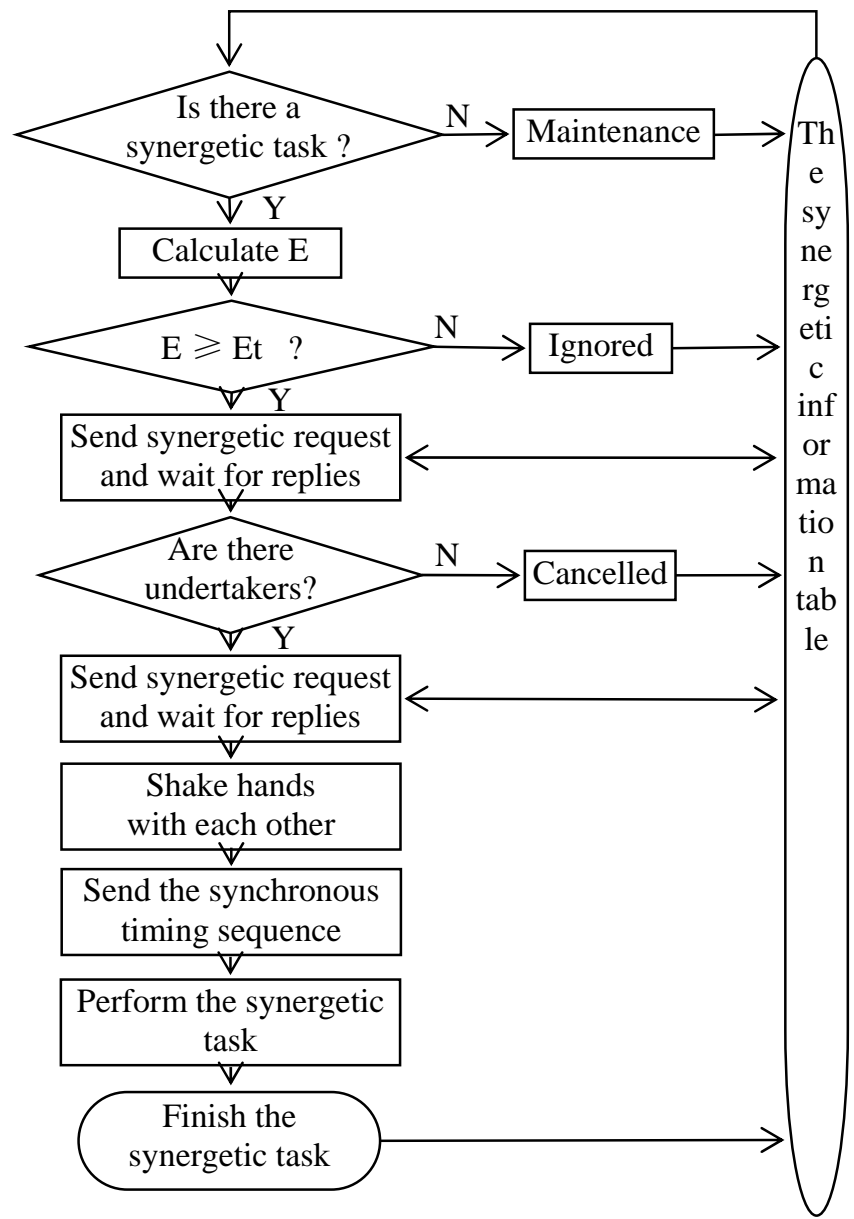

FIGURE II. THE SYNERGETIC PROCESS OF THE SPONSOR

If the PSC is invited to take party to a synergetic task, and it will also compute the synergetic discriminant value $\mathrm{E}$ according to the present situation, after receiving the synergetic request from the sponsor of the synergetic task. When the $\mathrm{E}$ is greater than Et, the synergetic task will be undertaken, and then the Handshaking signal will be replied, and the synergetic task will be done waiting for synchronous sequential joint implementation of the sponsor, otherwise the synergetic task will be ignored, see Fig. 3 .

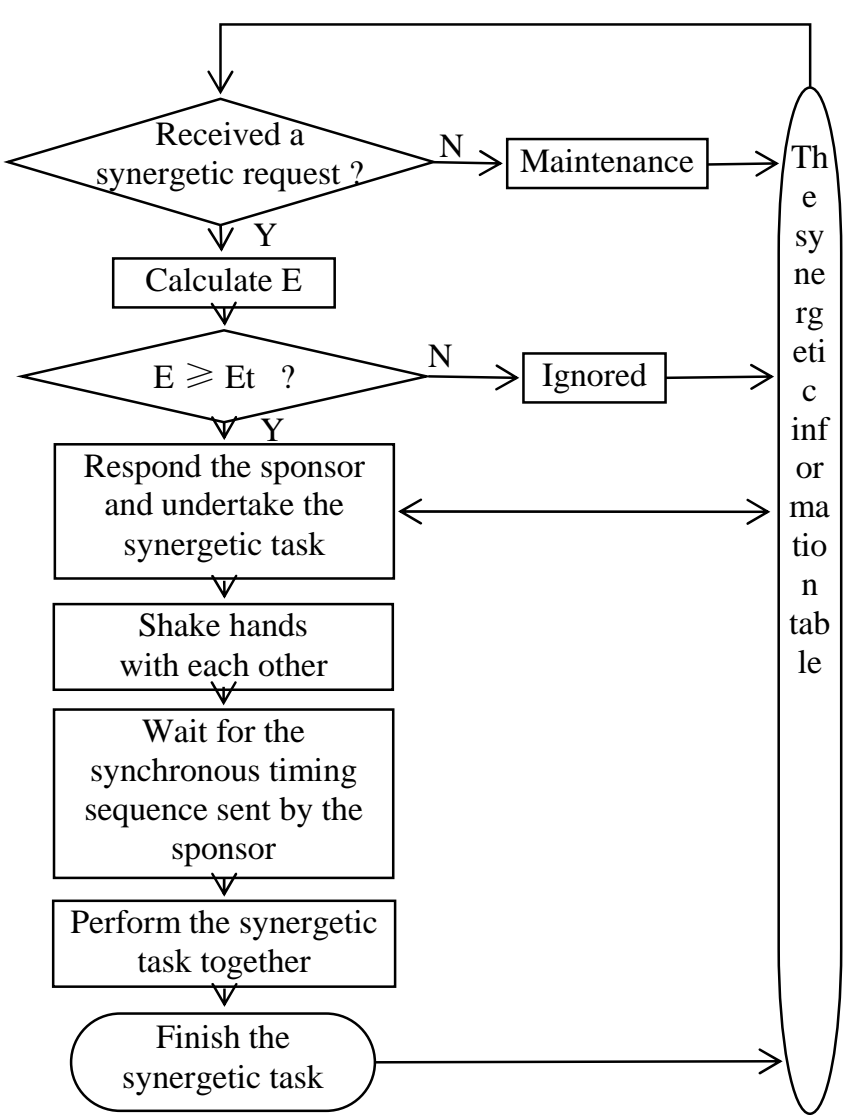

FIGURE III. THE SYNERGETIC PROCESS OF THE INVITEE

\section{The Concurrent Communication with Multiple Channels}

The communication is the key to PSC's ability to work together. If there is no or poor communication, the information transfer will be blocked and even cannot be achieved, and the synergetic tasks cannot be successfully delivered and implemented. The speed, reliability and accuracy of the communication have a direct impact on the efficiency and success or not of collaborative work with the PSC in the application system. Therefore, the importance of communication for PSC can be imagined.

The Concurrent Communication with Multiple Channels programs have been embedded in the Concurrent Multiple Communication Module. The communication effect, the signal strength and busy level of each wireless [3] and wired communication port are being monitored dynamically. If there is a amount of data need to be sent, especially the data volume is very large, the data will be automatically split and encryption in accordance with the rules, assigned to the best communication ports and links, and sent simultaneously in parallel to receiver. At the receiver, the data are decrypted, assembled and restored in accordance with the same rules.

\section{The Synergetic Information Code and PSC Programming}

In order to minimize the amount of communication data and improve the efficiency of communication and synergetic tasks to be executed, synergetic information is encoded beforehand. Based on the synergetic information code, some 
functional blocks and statements related to synergetic operation are constructed. The programming language of the original PLC is extended, and a PSC programming language and software tools are created. According to the specific application, the user program which contains the synergetic operations is described by programmer with this synergetic information code and PSC programming language, and then downloaded to PSC.

\section{CONCLUSION}

This PSC has been granted the patent of national invention patent, and can be applied to various automatic control systems. It can be applied not only to a single automatic control system and a DCS with multiple controllers, but also to a no master control system. If the PSC of a node fails, other PSC can spontaneously cooperate with each other to replace the PSC that losts its function. The reliability and fault tolerance of the application system will be improved greatly. At present, some of the very simple collaborative operations have been achieved only, and in the subsequent applications, the more synergetic functions will be constantly improved.

\section{ACKNOWLEDGMENT}

This research was financially supported by the Shandong Province key research and development plan (2015ZDZX10002 / 2015ZDXX0101G05), National key R \& D projects (2016YFD0705103 / 2017YFD0107200), Science and technology innovation project of Shandong Academy of Sciences(Shandong Institute of [2015]83).

\section{REFERENCES}

[1] Sun Xiang, Cheng Guanghe, Meng Qinglong, Synergetic Programmable Controller and Synergetic Method Thereof: CN102637028A[P]. 2012.

[2] Sung T. Analysis And Modeling Of Innovative Hybrid Plc-Wireless Cooperative System[J]. Sung Tae, 2010.

[3] Muniz A, Tsukamoto K, Kawahara K, et al. An Effectiveness Evaluation of PLC/WLAN Cooperative Transmission Scheme under Practical Network Environment[C]// International Conference on Network-Based Information Systems. IEEE Computer Society, 2014:438-443.

[4] Yongwei Tang, Chunmei Wang, Maoli Wang, Based on self-learning dictionary Circuit Board Fault Diagnosis Device, 2017 IEEE 2nd Advanced Information Technology, Electronic and Automation Control Conference, 2017, p2653-2657. 